\title{
Nicht die Jugendgewalt, sondern deren polizeiliche Registrierung hat zugenommen
}

\author{
- Ergebnisse einer Vergleichsstudie nach 25 Jahren
}

Dietrich Oberwittler und Tilman Köllisch

Der starke Anstieg der polizeilichen Tatverdächtigenziffern von Kindern und Jugendlichen in den letzten Jahren, insbesondere bei den Gewaltdelikten, wird von vielen Fachleuten auf ein verändertes Anzeige- und Registrierungsverhalten zurückgeführt. Allerdings gibt es nur wenige empirische Hinweise für diese Vermutung. Anhand einer lokalen Vergleichsstudie im Abstand von 25 Jahren präsentieren wir erstmals einen direkten Beleg für ein langfristiges verändertes Anzeigeverhalten bei Jugendgewalt. Wir interpretieren diesen Befund als Folge einer anhaltenden Tendenz zu einem sinstitutionalisierten Risikomanagement ‘ bei der Reaktion auf Verhaltensprobleme von Kindern und Jungendlichen und als Kehrseite des Verrechtlichungsprozesses von Kindheit.

Die Entwicklung der Jugendkriminalität im polizeilichen Hellfeld ...

S tatistiken der Jugendkriminalität haben die seltene Eigenschaft, dass sie scheinbar nur einen Trendverlauf kennen - einen nach oben gerichteten. Von kurzen Ruhepausen unterbrochen, zeigen die Zeitreihen der Polizeilichen Kriminalstatistik (PKS) über Jahrzehnte hinweg einen kontinuierlichen Anstieg der Jugendkriminalität in Deutschland an. Für Kriminologen gilt Jugendkriminalität daher als ,genuine Anstiegskriminalität (Walter, 2001, S.249). Auch das vergangene Jahrzehnt zeichnet sich durch einen starken Anstieg der Jugendkriminalität - insbesondere bei Gewaltdelikten aus, der jedoch in jüngster Zeit offenbar zu einem (vorläufigen) Ende gekommen ist.

Diese Entwicklung lässt sich anhand zweier Graphiken knapp beschreiben. Zwischen 1987 und 2003 ist die Tatverdächtigenbelastungsziffer (TVBZ) der Kinder ( 8 bis 13 Jahre), Jugendlichen (14 bis 17 Jahre) und Heranwachsenden (18 bis 20 Jahre) um $80 \%$ bis $100 \%$ angestiegen, während die der Erwachsenen nur um 20\% zugelegt hat (siehe Abbildung 1). ${ }^{1}$ Besonders stark war die Zunahme bei den Betäubungsmittel- und Gewaltdelikten, und hier jeweils umso stärker, je jünger die Tatverdächtigen waren. So hat sich die TVBZ der Kinder bei Gewaltdelikten in den letzten 15 Jahren mehr als vervierfacht, die der Jugendlichen beinahe verdreifacht, während sie bei den Erwachsenen >nur $<$ um 50\% angestiegen ist. Es fällt auf, dass beide Deliktgruppen besonders anfällig für Veränderungen des Kontroll- und Anzeigeverhalten von Polizei bzw. Opfern sind. Dies legt die Vermutung nahe, dass der Anstieg der registrierten Kriminalität zumindest teilweise auf eine verstärkte Erfassung von Jugenddelinquenz durch die Polizei erklärt werden kann. Der Anstieg bei den Diebstahlsdelik- ten war demgegenüber wesentlich geringer, bei schwerem Diebstahl ist bis auf die Altersgruppe der Jugendlichen sogar ein Rückgang um -10\% bis $-30 \%$ festzustellen. Abbildung 2 illustriert anhand der Zeitreihen der Gewalt-TVBZ ab 1973 in Baden-Württemberg, dass der Anstieg der registrierten Gewaltkriminalität vollständig auf die jüngeren Altersgruppen beschränkt ist. Während in den 1970er Jahren die Jugendlichen bei der Gewaltbelastung noch deutlich unter den Heranwachsenden lagen, und die Belastungsziffer der Kinder nahe Null lag, befinden sich Jugendliche und Heranwachsende nun gleichauf, und die Kinder hatten 2001 sogar eine höhere Belastungsziffer als die Erwachsenen. Demnach sind die polizeilich registrierten Gewalttäter tat-

Abbildung 1: Veränderung der Tatverdächtigenbelastungsziffern nach Deliktarten, Deutschland 1987 bis 2003 (in \%, nur deutsche Tatverdächtige)

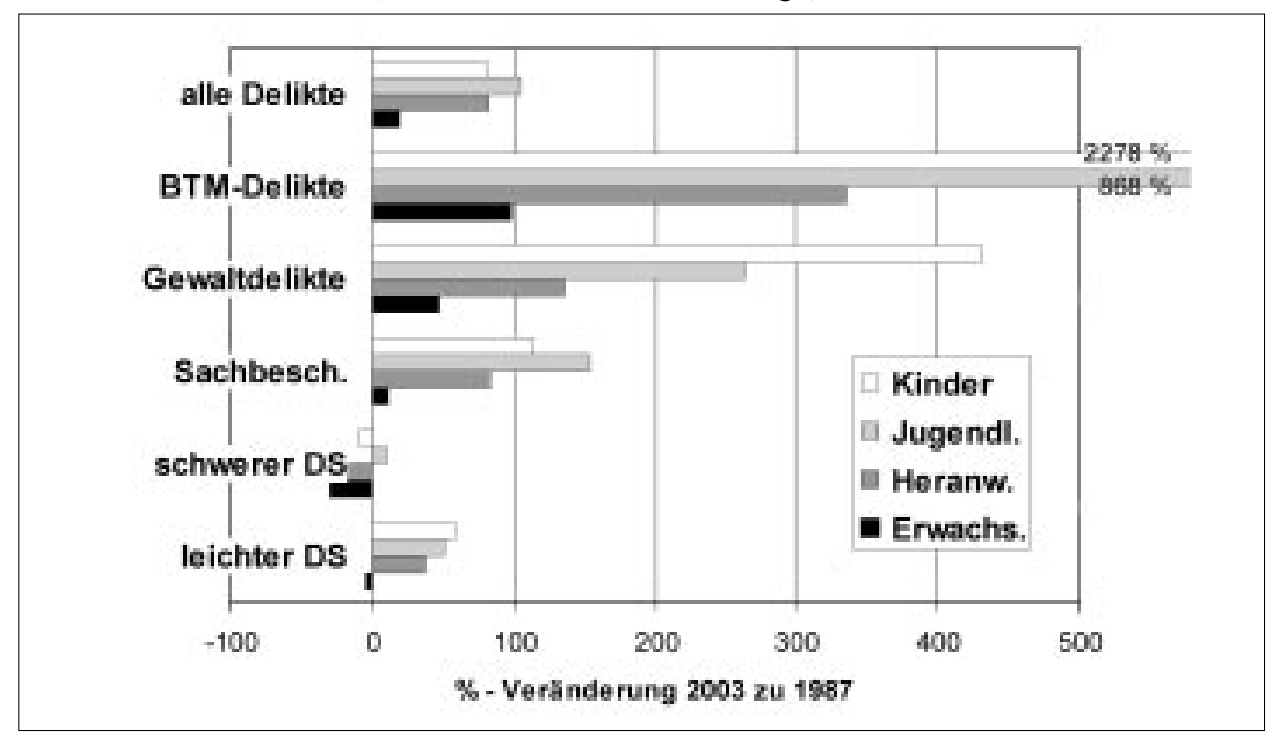

Quelle: Bundeskriminalamt, PKS-Zeitreihen 1987 bis 2003, Tabelle 40, eigene Berechnung sächlich >immer jünger geworden, wie es in den Medien so griffig formuliert wird.

Wie bei früheren Anstiegswellen auch, ist die jüngste Welle von einer gesteigerten Beunruhigung der Öffentlichkeit über >die Jugend ihr Fehlverhalten begleitet worden und hat den Forderungen nach Verschärfungen des Jugendstrafrechts Auftrieb gegeben. Die Reaktionen der Experten fielen differenzierter aus. Einige Fachleute gestanden der PKS zu, Veränderungen des statsächlichen ‘ delinquenten Verhalten von Jugendlichen wenigstens der Tendenz nach richtig wiederzugeben, und suchten nach den sozialen Ursachen dieser Entwicklung (Pfeiffer \& Ohlemacher 1995). Andere sahen sich durch den starken Anstieg in ihrer Position bestätigt, dass 
Abbildung 2: Entwicklung der Tatverdächtigenbelastungsziffern bei Gewaltdelikten, Baden-Württemberg 1973 bis 2003



Quelle: LKA Baden-Württemberg, Polizeiliche Kriminalstatistik, verschiedene Jahrgänge; LKA Baden-Württemberg, Jahresbericht Jugendkriminalität und Jugendgefährdung, verschiedene Jahrgänge: eigene Berechnungen

Kriminalstatistiken viel über die Arbeit der Polizei und wenig über die Delinquenz von Jugendlichen aussagen, und dass aus einer Zunahme in der Statistik nicht auf eine tatsächliche Zunahme des strafbaren Verhaltens geschlossen werden könne (Walter 1996).

\section{Die Entwicklung der Jugendkriminalität im polizeilichen Dunkelfeld}

Seit diese Positionen in der zweiten Hälfte der 90er Jahre kontrovers diskutiert wurden, hat die ,Dunkelfeldforschung in Deutschland mit einer Reihe von Schulbefragungen, bei denen Jugendliche zu ihrer Delinquenz befragt werden, einen erheblichen Aufschwung erfahren. Dies hat es zumindest ansatzweise ermöglicht, von der PKS unabhängige Informationen über das Ausmaß und die Entwicklung der Jugenddelinquenz zu gewinnen, die auch indirekte Hinweise auf mögliche Veränderungen des Anzeigeverhaltens geben können. Denn es ist unumstritten, dass das Anzeigeverhalten der Opfer insbesondere bei Gewaltdelikten einen entscheidenden Einfluss auf das Verhältnis von Hell- und Dunkelfelddelinquenz und damit auf die Entwicklung der PKS hat. Da der größte Teil potenziell strafbarer Handlungen von Jugendlichen im Dunkelfeld verbleibt, kann schon eine relativ geringe $\mathrm{Zu}$ nahme der Anzeigen einen spürbaren Anstieg der polizeilich registrierten Jugendkriminalität bewirken.

Da jedoch in Deutschland anders als beispielsweise in den Niederlanden oder Großbritannien keine regelmäßigen, national-repräsentativen Täter- und Opferbefragungen durchgeführt werden, beruhen alle bisherigen Erkenntnisse auf zeitlich beschränkten Lokal- oder Regionalstudien, die schwerlich verallgemeinerbar sind. So wurde bei einer Schulbefragung zur selbstberichteten Delinquenz, die 1995 im Raum Kassel durchgeführt wurde, der gleiche Fragebogen verwendet wie bei einer Befragung in Bielefeld im Jahr 1973 (Tillmann u. a. 1999). Dabei zeigte sich, dass sich der Anteil der gewalttätigen SchülerInnen von 5\% 1972 auf 12,8\% 1995 mehr als verdoppelt hatte. Bei Sachbeschädigungsdelikten fiel der Anstieg der Täterrate von 11\% auf 16,9\% wesentlich geringer aus, und bei Diebstahlsdelikten war die Zunahme von 7\% auf 10,7\% am geringsten. Bei einer Wiederholungsbefragung an zwei Nürnberger Hauptschulen im Jahr 1995, die ebenfalls 1973 schon einmal an einer Schulbefragung teilgenommen hatten, stellen Lösel $u$. a. (1998) eine Zunahme der Delinquenz fest, die sich weniger in einer Erhöhung der Anzahl der Täter, sondern eher in der Deliktshäufigkeit in der Gruppe der >Intensivtäter ‘ ausdrückte. Die größte Vergleichsstudie, die 1988 und 1996 an nordrhein-westfälischen Schulen durchgeführt wurde, deutet ebenfalls auf einen Anstieg der Jugenddelinquenz im Dunkelfeld hin (Mansel \& Hurrelmann 1998). Die Rate der Gewalttäter hat sich demnach bei Jungen von $45,2 \%$ auf $58 \%$ und bei Mädchen von 27,3\% auf 38,2\% erhöht; die Zahl der Mehrfachtäter stieg von 6,6\% auf 9,7\%, was einer Zunahme um knapp 50\% entspricht. Bei den Eigentumsdelikten verdoppelte sich die Zahl der Mehrfachtäter sogar von 5,0\% auf $10,0 \%$.

Bei der Bewertung dieser Vergleichsstudien ist $\mathrm{zu}$ berücksichtigen, dass auch ein veränderter Gewaltbegriff und ein verändertes Antwortverhalten der befragten Jugendlichen Einfluss auf die Befragungsergebnisse haben könnten. Men- zel \& Peters (1998) haben darauf hingewiesen, dass insbesondere eine erhöhte Sensibilität gegenüber Gewalt im Zuge der öffentlicher Diskussionen dazu führen könne, dass Schüler sihr Handeln [heute] häufiger als Gewalt verstehen als früher. Gegen diese These sprechen jedoch unter anderem sinkende Gewaltraten, die trotz der anhaltenden Gewaltdiskussion in einigen aktuellen Delinquenz- und Opferbefragungen gemessen wurden. So ergibt der regelmäßig durchgeführte National Crime Survey in den USA seit 1994 - in Übereinstimmung mit der Entwicklung der polizeilich registrierten Kriminalität - eine sinkende Gewaltbelastung von Jugendlichen (Lynch, 2002). Es gibt bislang wenig Hinweise darauf, dass sich eine veränderte Bewertung des eigenen Verhaltens stärker auf die Befragungsergebnisse auswirkt als die Veränderung dieses Verhaltens selbst.

Insgesamt kann man auf der Basis dieser Vergleichsstudien vorsichtig annehmen, dass die Jugenddelinquenz im Dunkelfeld in den letzten zehn bis zwanzig Jahren zwar zugenommen hat, dass diese Zunahme aber nicht so groß ausfällt wie dies im polizeilichen Hellfeld erscheint. Wenn die Jugend nicht wesentlich krimineller geworden ist - so wird aus diesen Ergebnissen indirekt gefolgert - dann muss eine Zunahme der Anzeige- und Registrierungswahrscheinlichkeit für den Anstieg der Kriminalstatistik verantwortlich sein. Jedoch wurde in keiner der längerfristigen Vergleichsstudien direkt danach gefragt, ob und wie oft die Jugendlichen als Täter mit der Polizei in Kontakt geraten sind, oder ob sie als Gewaltopfer selbst eine Anzeige erstattet haben.

\section{Ergebnisse einer wiederholten Dunkelfeld- studie nach 25 Jahren}

Genau dies ist der Vorteil einer neuen Vergleichsstudie, über die wir in diesem Beitrag berichten wollen. Es handelt sich dabei um einen Vergleich zweier Dunkelfeldbefragungen männlicher Jugendlicher in der südbadischen Kleinstadt Emmendingen, die im Abstand von 25 Jahren 1973 und 1999 - vom Max-Planck-Institut für Strafrecht Freiburg durchgeführt wurden (Villmow \& Stephan 1983; Oberwittler \& Würger 1999). Da in beiden Befragungen nicht nur nach der Delinquenz, sondern auch nach den damit verbundenen Polizeikontakten (sowohl aus der Täter- wie aus der Opferperspektive) gefragt wurde, können erstmals auch langfristige Veränderungen in der Anzeigewahrscheinlichkeit und im Registrierungsrisiko direkt untersucht werden. Allerdings basieren auch diese Befragungen auf relativ kleinen und lokal begrenzten Stichproben von weniger als 200 Befragten, so dass die Ergebnisse vorsichtig interpretiert werden sollten. Insofern sich zwischen 1973 und 1999 Änderungen in der methodischen Durchführung der Befragungen ergeben haben, sollten diese eher 
Abbildung 3: Selbstberichtete Delinquenz und Polizeikontakte männlicher Jugendlicher in der südbadischen Stadt Emmendingen im Zeitvergleich (1973 und 1999)



Quelle: Erhebungen des Max-Planck-Instituts für ausländisches und internationales Strafrecht Freiburg (Köllisch \& Oberwittler 2004)

zu einer Überschätzung der Delinquenz in der jüngsten Befragung führen. ${ }^{2}$

Die wichtigsten Ergebnisse des Vergleichs lassen sich aus Abbildung 3 ablesen. Nur bei den Betäubungsmitteldelikten gab es im Dunkelfeld eine erhebliche (und statistisch signifikante) $\mathrm{Zu}$ nahme der Täter, während die Täterrate bei Diebstahl und Sachbeschädigung nur moderat gestiegen, und bei Gewaltdelikten sogar von ca. $11 \%$ auf $9 \%$ leicht gefallen ist. Ein sehr ähnliches Bild ergibt sich auch, wenn man zusätzlich die Delikthäufigkeit der Täter berücksichtigt. Auch aus der Opferperspektive berichten die 1999 befragten Jugendlichen nicht häufiger über Gewalterfahrungen als 1973. Die Jugendgewalt hat demnach in der untersuchten Stadt in den letzten 25 Jahren nicht zugenommen.

Ganz anders ist dagegen in der Zeitspanne von 25 Jahren die Entwicklung des Risikos von Polizeikontakten verlaufen, das wir als den Anteil der Befragten mit Polizeikontakten an den Tätern definieren. Aufgrund sehr niedriger Fallzahlen ist hier kaum mit hochsignifikanten Ergebnissen zu rechnen. Dennoch zeigt sich, dass insbesondere bei Gewaltdelikten, aber auch beim Diebstahl die Wahrscheinlichkeit eines Polizeikontaktes für die Täter signifikant zugenommen haben, während bei Sachbeschädigungsund Betäubungsmitteldelikten keine bedeutsamen Veränderungen aufgetreten sind. Während 1973 keiner der Befragten, die ein oder mehrere Gewaltdelikte angegeben haben, über einen Polizeikontakt berichten, ist es 1999 jeder Dritte. Bei Diebstahlsdelikten stieg die Rate der Polizeikontakte von $7 \%$ auf $26 \%$ an. Auch aus der Opferperspektive wird dieser Trend wiederum bestätigt: Bei Gewaltdelikten verdoppelte sich die Anzeigerate der Opfer von 14\% auf 30\%, bei Diebstahlsdelikten kletterte sie von $22 \%$ auf $36 \%$. Demgegenüber berichten Befragte, die
Sachbeschädigungen und illegalen Drogenkonsum zugegeben haben, auch 1999 nicht über Polizeikontakte. Dies widerspricht zwar dem Anstieg der Zahlen in der PKS, jedoch ist $\mathrm{zu}$ bedenken, dass bei beiden Delikten der Anteil der ins Hellfeld gelangten Fälle immer noch sehr klein ist, und es wahrscheinlich größerer Stichproben bedarf, um Jugendliche zu finden, die solche Polizeikontakte hatten.

Die Ergebnisse unserer Studie sprechen für die Vermutung, dass der enorme Anstieg der regis-

Abbildung 4: Indikatoren des institutionellen Risikomanagements bei Kindern und Jugendlichen

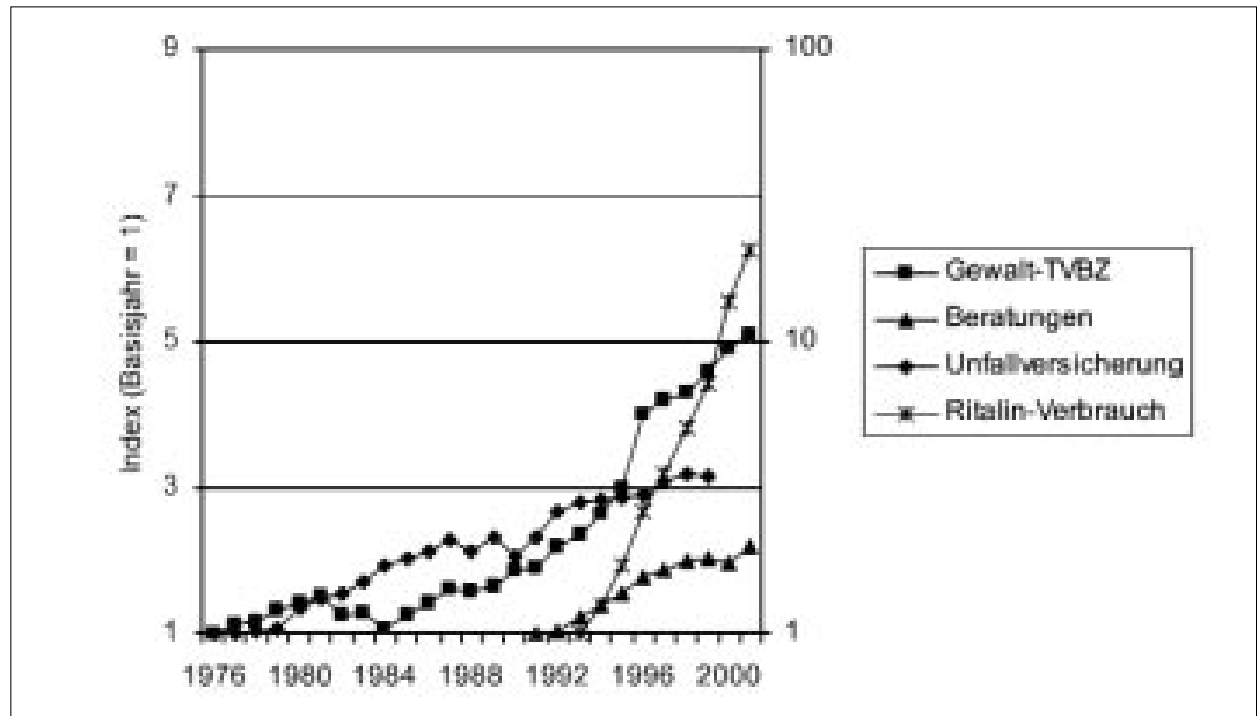

Gewalt-TVBZ: siehe Abbildung 2 Wesentlichen auf die >Aufhellung eines weitgehend stabilen Dunkelfelds durch vermehrte Anzeigen zurückzuführen ist. Bei Diebstahlsdelikten scheint sich dagegen sowohl das Dunkelfeld vergrößert als auch die Anzeigewahrscheinlichche Zunahme des Konsums illegaler Drogen entin trale für gesundheitliche Aufklärung (2001, schen Problemen für die Validität des Zeitvergeiches. lung der Jugenddelinquenz und ihrer Kontrolle ergeben kann. Punktuelle Studien können keinen Ersatz sein für regelmäßige, national-reprälinquenz und Viktimisierung von Jugendlichen die es in Deutschland nicht gibt. Aber nur so ließen sich die divergierenden Trends im Dunkelund Hellfeld voneinander trennen. Dies könnte zu einer Versachlichung der öffentlichen Diskusauf die Ursachen und Folgen der zunehmend formellen Sozialkontrolle des Verhaltens von Kindern und Jugendlichen verlagern. für Arzneimittel und Medizinprodukte, Mitteilung vom 8.08.2002)

Unfallversicherungen: Versicherungsdichte von Minderjährigen in Deutschland (Quelle: Gesamtverband der Deutschen Versicherungswirtschaft e.V., Mitteilung vom 9.08.2002)

Beratungen: Anzahl der beendeten Erziehungs-/Familien-, Jugend- und Suchtberatungen (Altersgruppe 15-19 Jahre) in Baden-Württemberg (Quelle: Statistisches Landesamt Baden-Württemberg) 


\section{Sozialer Wandel des Risikomanagements bei Kindern und Jugendlichen}

Dafür wollen wir abschließend eine Interpretation anbieten, die auf den Verlust informeller Konfliktregelungskompetenzen und die Verrechtlichung der Familie in unserer Gesellschaft abhebt. Einige Indizien sprechen dafür, das der seit der Industrialisierung stattfindende Prozess der Verlagerung 'privater Lebensbereiche aus der Familie in eine öffentliche Sphäre noch nicht abgeschlossen ist und insbesondere durch die `nachholende Individualisierung ‘ der Frauen angetrieben wird (Beck-Gernsheim, 1998). Auf der Mikroebene sind dafür der weitere Anstieg von Müttererwerbstätigkeit, Ehescheidungen und Alleinerziehendenhaushalten Indizien, während auf der Makroebene weiterhin Tendenzen einer Verrechtlichung des Eltern-Kind-Verhältnisses, zum Beispiel in der UN-Kinderrechtskonvention, der Neuregelung des Sorgerechts oder im Verbot der elterlichen Züchtigung, sichtbar werden. Eine zunehmende formelle Sozialkontrolle von Kindern und Jugendlichen erscheint in diesem Kontext als eine negative Kehrseite dieses Verrechtlichungsprozesses, auch wenn er grundsätzlich durch ein erhöhtes Schutzbedürfnis gegenüber Kindern motiviert sein mag. Es scheint jedoch, dass auf Probleme im Aufwachsen von Kindern und Jugendlichen ganz generell zunehmend mit Formen eines professionellen ,Risikomanagements' reagiert wird. Eine solche allgemeine Tendenz lässt sich in einer Reihe von Feldern aufzeigen, wobei unsere in Abbildung 4 dargestellten Zeitreihen eher impressionistischen Charakter haben. Neben der nochmals dargestellten Zunahme der registrierten Gewaltrate der Jugendlichen in Baden-Württemberg verläuft der Anteil der Minderjährigen in Deutschland, für die Unfallversicherungen abgeschlossen wurden, fast zwei Jahrzehnte lang weitgehend parallel; die Versicherungsdichte stieg von 14\% im Jahr 1976 auf rund 45\% im Jahr 1999. Die Zeitreihe der Beratungsgespräche in baden-württembergischen Erziehungs- und Suchtberatungsstellen für die Altersgruppe 15 bis 19 Jahre beginnt erst 1991, weist jedoch bis 2001 einen Anstieg um mehr als das Doppelte auf. Schließlich zeigt der Verbrauch des Wirkstoffs Methylphenidat (Handelsname Ritalin), der zur medikamentösen Behandlung von Aufmerksamkeitsdefizits- und Hyperaktivitätssyndromen bei Kindern und Jugendlichen eingesetzt wird, eine außerordentliche Steigerung von $34 \mathrm{~kg}$ im Jahr 1993 auf 694 $\mathrm{kg}$ im Jahr 2001, die wir in der Abbildung 3 logarithmisch (rechte Skala) dargestellt haben. Ohne die spezifischen Hintergründe und Probleme dieser Zahlen im Einzelnen zu thematisieren glauben wir doch, dass sie etwas Gemeinsames messen, nämlich die wachsende Bedeutung von professionellen und institutionellen Formen des Umgangs mit Verhaltensproblemen von Kindern und Jugendlichen. Das `Management< von spezifischen sozialen Risiken, die mit dem Sozia- lisationsprozess verbunden sind, wird offenbar zunehmend von professionellen und institutionellen Akteuren übernommen, zu denen eben auch die Polizei gehört. Es ist daher kaum anzunehmen, dass sich dieser Trend wieder umdrehen könnte.

Welche Bedeutung die Zunahme der polizeilichen Registrierungen für die betroffenen Jugendlichen selbst und für das Funktionieren der staatlichen Sozialkontrolle hat, kann hier zum Schluss nur kurz angedeutet werden. Der alte Lehrsatz der Kriminologie, dass abweichendes Verhalten im Jugendalter eine Normalität ist, erhält dadurch eine neue Bedeutung. In Zukunft werden ca. $40 \%$ bis $50 \%$ statt der heute üblichen $25 \%$ der männlichen Jugendlichen polizeiauffällig geworden sein, bis sie 20 Jahre alt sind. Während man in der Denktradition des Labeling Approach befürchten könnte, dass eine polizeiliche Registrierung Stigmatisierungseffekte mit negativen Auswirkungen für die betroffenen Jugendlichen auslösen könnte, erscheinen uns die oft zitierten Überlegungen des Soziologen Heinrich Popitz von der >Präventivwirkung des Nichtwissens und deren Gefährdung durch eine zu rigorose Aufdeckung des Dunkelfelds ebenso plausibel. Popitz wies auf die Abhängigkeit der Wirksamkeit von Sanktionen von ihrer sparsamen Anwendung hin: »Etwas, das beinahe jedem reihum passiert, gilt nicht mehr als diskriminierend«(Popitz, 1968, S.17).

\section{Literatur:}

Beck-Gernsheim, E. (1998). Was kommt nach der Familie? Einblicke in neue Lebensformen. München: Beck.

Köllisch, T. \& Oberwittler, D. (2004). Sozialer Wandel des Risikomanagements bei Kindern und Jugendlichen. Eine Replikationsstudie zur langfristigen Zunahme des Anzeigeverhaltens. Zeitschrift für Soziologie der Erziehung und Sozialisation 24(1), 49-72.

Lösel, F., Bliesener, T. \& Averbeck, M. (1998). Hat die Delinquenz von Schülern zugenommen? Ein Vergleich im Dunkelfeld nach 22 Jahren DVIJ-Journal, 9, 115 -125.

Lynch, J.P. (2002). Trends in Juvenile Violent Offending: An Analysis of Victim Survey Data. Washington, D.C.: U.S. Department of Justice, Bureau of Justice Statistics.

Mansel, J. \& Hurrelmann, K. (1998). Aggressives und delinquentes Verhalten Jugendlicher im Zeitvergleich. Befunde der >Dunkelfeldforschung aus den Jahren 1988, 1990 und 1996. Kölner Zeitschrift für Soziologie und Sozialpsychologie, 50, 78-109.

Menzel, B. \& Peters, H. (1998). »Self-Reports« taugen wenig für objektive Vergleiche. Kölner Zeitschrift für Soziologie und Sozialpsychologie, 50, 560-564.

Oberwittler, D. \& Würger, M. (1999). Emmendinger Schülerbefragung zur Jugenddelinquenz 1999.

Pfeiffer, C. \& Ohlemacher, T. (1995). Anstieg der (Gewalt-)Kriminalität und der Armut junger Menschen. In S. Lamnek (Hrsg.), Jugend und Gewalt. Devianz und Kriminalität in Ost und West (S.259-276). Opladen: Leske + Budrich.
Popitz, H. (1968). Über die Präventivwirkung des Nichtwissens. Dunkelziffer, Norm und Strafe. Tübingen: J.C.B. Mohr.

Villmow, B. \& Stephan, E. (1983). Jugendkriminalität in einer Gemeinde: Eine Analyse erfragter Delinquenz und Viktimisierung sowie amtlicher Registrierung. (Kriminologische Forschungsberichte aus dem Max-Planck-Institut für ausländisches und internationales Strafrecht Freiburg / Bd. 6.). Freiburg: Max-Planck-Institut.

Walter, M. (1996). Kriminalpolitik mit der polizeilichen Kriminalstatistik? Artikulation eines Unbehagens über den derzeitigen Kurs der Deutschen Jugendgerichtsvereinigung. DVIJJournal, 7, 209-214

Dietrich Oberwittler ist Soziologe und z.Zt. Marie Curie Visiting Scholar am Institute of Criminology, University of Cambridge (GB).

Tilmann Köllisch ist Soziologe und wissenschaftlicher Mitarbeiter am Lehrstuhl für Kriminologie, Jugendstrafrecht und Strafvollzug an der Universität Giessen.

Während der Durchführung der Studie arbeiteten beide Autoren in der Kriminologischen Forschungsgruppe am Max-Planck-Institut für ausländisches und internationales Strafrecht Freiburg i.Br.

Eine erweiterte Fassung dieses Aufsatzes ist in Band 24 (2004) der Zeitschrift für Soziologie der Erziehung und Sozialisation erschienen.

\section{Fußnoten:}

1 Diese Zahlen beziehen sich nur auf deutsche Tatverdächtige, da das Bundeskriminalamt wegen der Problematik der nicht zur Wohnbevölkerung zählenden Tatverdächtigen keine Belastungsziffern für ,ausländische' Tatverdächtige und dementsprechend auch keine Gesamt-Belastungsziffern veröffentlicht.

2 Für eine ausführliche Beschreibung der methodischen Aspekte siehe Köllisch und Oberwittler (2004). 